\title{
Introduction to the Issue on Advanced Signal Processing in Microscopy and Cell Imaging
}

M ICROSCOPY imaging, including fluorescence microscopy and electron microscopy, has a prominent role in life science and medical research. During the past two decades, biological imaging has undergone a revolution by way of the development of new microscopy techniques that allow the visualization of tissues, cells, proteins and macromolecular structures at all levels of resolution, physiological states, chemical composition and dynamic analysis. Thanks to recent advances in optics, digital sensing technologies and labeling probes (i.e., XFP-Colored Fluorescence Protein), we can now visualize subcellular components and organelles at the scale of a few nanometers to several hundreds of nanometers. As a result, fluorescent microscopy has become the workhorse of modern biology. Further technological advances include structured and coherent light sources, faster and more sensitive detectors, smaller and more specific molecular probes and automation processes for image acquisition. Additionally, there is a push towards multimodal imaging in order to gather complementary information such as the concentration of fluorophores at various wavelengths and the refractive index of a given sample (phase imaging).

With all the advances in technology for microscopy, many of the existing roadblocks in biological and medical research at the microscopic level are problems of signal processing. The need to analyze multidimensional images acquired with high spatial and temporal resolution microscopy poses new challenges for researchers in image processing and image analysis. A salient aspect of microscopy data is the highly variable nature of biological objects (cells, organelles, single molecules, etc.) as well as the range of scales. A second aspect is the dynamic nature of the data and the complexity of biological processes involving many entities (chromosomes, vesicles, membrane fusion and budding). Therefore, dedicated efforts are necessary to develop and integrate cutting-edge approaches in image processing and optical technologies to push the limits of the instrumentation and to analyze and quantify the large amount of data being produced. Once the numerical processing is part of the imaging loop, such processing may actually drive the imaging. A dramatic example of this shift in paradigm is super-resolution localization microscopy (PALM and STED), which was rewarded with the 2014 Nobel Prize in Chemistry.

This special issue is devoted to advances in microscopy image processing with a special focus on mathematical imaging and algorithms design. It covers a wide scope of applications and challenges that are specific to the field, from image reconstruction, segmentation, and classification to object tracking.

Methods that are used for reconstructing microscopy images share commonalities with those deployed in medical imaging - the recent push is also towards "compressed sensing" and the use of sparsity to guide the reconstruction process. This issue includes several papers that apply sparsity-driven models and modern optimization techniques for improving image reconstruction. Arildsen, Oxvig, Pedersen, Østergaard,

Digital Object Identifier 10.1109/JSTSP.2015.2511299 and Larsen present an approach based on sparse approximation and interpolation to reconstruct images in atomic force microscopy. They propose to reduce the critical scanning time and probe-specimen interaction through the use of a sparse (raster and spiral) sampling pattern. Song and Horowitz revisit the classical subtomogram averaging problem in cryo-electron tomography by reformulating it as a low-rank matrix recovery problem subject to constraints on the misfit of to the radon measurements. The proposed approach does not require prior information, such as the number of classes or references of the target structures. It also addresses the missing wedge issue by formulating tomographic sensing operators at each projection angle. The idea of Fortun, Guichard, Chu, and Unser is to combine multiple views of identical specimens obtained at different orientations in order to overcome the intrinsic axial resolution limit of fluorescence microscopy. To that end, they develop a joint deconvolution-and-multiview reconstruction framework that relies proximal splitting. They present preliminary results of super-resolution 3D reconstruction, as proof of concept.

Images in light microscopy are typically blurred and noisy because of the diffraction-limited and photon-limited nature of the data. Specific algorithms are required to restore information and the special issue includes two such contributions. Ponti, Helou, Ferreira, and Mascarenhas introduce a new approach for image deconvolution that relies on constraint sets and sub-gradient projections. The Richardson-Lucy iterations within the POCS framework are interpreted as a gradient iteration allowing constraints to be enforced during restoration of wide-field and confocal images. Tofighi, Yorulmaz, Köse, Yildirim, Çetin-Atalay, and Çetin propose of novel approach for blind deconvolution where the constraints are specified in terms of two closed convex sets convex sets. First, the set of images with a prescribed Fourier Transform phase is used as constraint set; then, a second set called the Epigraph Set of Total Variation (TV) is used to automatically estimate an upper-bound on the TV value of a given image. Both are used as components of iterative microscopic image deblurring algorithms.

A standard paradigm for solving image reconstruction problems is to impose a statistical or regularization prior such as "total variation", which is now used routinely for deconvolution. It is expected that the use of better models should improve reconstruction quality. Lenz investigates the generalized extreme Pareto distribution (GPD). He relies on this probabilistic modeling to construct a sharpness function, which is then used to drive an autofocus algorithm for microscopy. Gong and Sbalzarini exploit the statistical distribution of the gradient of the image to specify a data-driven regularizer that can be used for solving a variety of inverse problems in light microscopy. The prior, which is learned from a collection of natural-scene images, then takes the familiar form of a variational energy.

The more advanced methodologies for cell classification are based on machine learning techniques and/or statistical modeling. Xu, Megjhani, Trett, Shain, Roysam, and Han present a general non-parametric Bayesian framework for the classification of arbor morphologies. Their scheme automatically iden- 
tifies the number of classes even if they are not clearly separated, as is the often case with complex biological datasets. To address the problem of cell classification in histopathology, Kang, Yoo, and Na propose a deep probabilistic architecture referred to the tree-structure sum-product network (t-SPN). It is trained using the maximum margin criterion and a 12 regularization to enhance generalization. The method exploits high-pass and Laplacian-of-Gaussian (LoG) filtering and compares favorably with conventional convolution neural networks on benchmark datasets.

Image segmentation remains a challenging problem in $3 \mathrm{D}$ microscopy due to the difficulty to separate closely packed cells, to detect nuclei or delineate cell membranes. The standard methodologies include level sets, active contours and watershed methods. In order to segment nuclei in 3D tissue images, Nandy, Chellappa, Kumar, and Lockett propose a novel seed-detection method followed by a graphcut. Their seed detection utilizes a robust model-based 2D slice-by-slice segmentation followed by a suppression of points that are not local maxima. Toutain, Elmoataz, Desquesnes, and Pruvot address the problem of $3 \mathrm{D}$ cell segmentation in confocal microscopy within a unifying graphical framework that combines filtering, image segmentation and classification. Their formulation involves partial derivative equations (PDE) on weighted graphs (graph p-Laplacian). Jonic and Sorzano propose a novel algorithm to convert a 3D transmission electron microscopy density volume into a granulated model by controlling the volume approximation error. This granularization method is appropriately developed to study macromolecular dynamics.

The use of time-lapse video-microscopy to capture dynamics of various biological objects has significantly increased in recent years. This special issue includes four papers on this topic. Sadanandan, Baltekin, Magnusson, Boucharin, Ranefall, Jaldén, Elf, Wälby present a fast and robust method for segmenting and tracking E. coli cells over time. It includes a quality control and refinement step to correct errors, which is crucial to perform well on phase contrast microscopy images. The algorithm of Chen, Zhao and Yan for tracking cells in 4D data during C. Elegans embryogenes is based on probabilistic relaxation labeling. The tracking is formulated as a non-rigid point matching problem. Their method has a fast parallel implementation, which can provide a significant advantage for large-scale image analysis. Schlangen, Franco, Houssineau, Pitkeathly, Clark, Smal, and Rickman adopt a Bayesian formulation to track moving object with different motion behaviors and to estimate sensor drift. A probability hypothesis density (PHD) filter with classification is combined with a particle filter to provide a joint estimation of the sensor movement with respect to the monitored sample and the intracellular motion of molecular structures in photo-activated localization microscopy (PALM). Pécot, Kervrann, Salamero, and Boulanger address the problem of traffic analysis at the sub-cellular level in 2D-3D fluorescence microscopy. They obtain an estimate of particle flux based on the global minimization of an energy functional with sparse constraints. The interest of their approach is that it lies in-between individual object tracking and dense motion estimation.

As Guest Editors of this special issue, we would like to thank all authors who submitted their manuscripts to this issue. We also thank the reviewers for their careful and conscientious reviews and timely responses. Without their efforts, it would have been impossible to prepare this issue. Finally, we express our appreciation to the IEEE JSTSP editorial board and the editorial staff for their help and support to facilitate the process of editing of this special issue.

\section{Charles Kervrann, Guest Editor \\ Serpico Project-Team Inria \\ Centre de Rennes-Bretagne Atlantique \\ 35042 Rennes, France}

Scott T. Acton, Guest Editor

Department of Electrical and Computer Engineering

University of Virginia

Charlottesville, VA 22904 USA

Jean-Christophe Olivo-Marin, Guest Editor
Quantitative Image Analysis Unit
Institut Pasteur
75015 Paris, France

Carlos Óscar Sánchez Sorzano, Guest Editor

National Center of Biotechnology (CSIC)

28049 Madrid, Spain

Michael Unser, Guest Editor

Biomedical Imaging Group

Swiss Federal Institute of Technology Lausanne

CH-1015 Lausanne, Switzerland

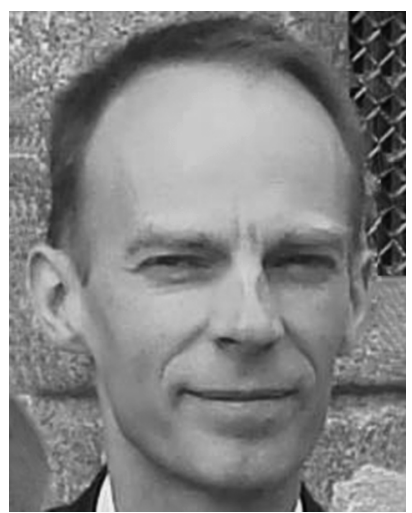

Charles Kervrann received the M.Sc. (1992), the Ph.D. (1995) and the HDR (Habilitation à Diriger des Recherches, 2010) in Signal Processing and Telecommunications from the University of Rennes 1, France. From 1997 to 2010, he was researcher at the INRA Applied Mathematics and Informatics Departement (1997-2003, Jouy-en-Josas, France) and he joined the VISTA Inria research group in 2003 (Rennes, France). In 2010, he was appointed to the rank of Research Director, Inria Research Centre in Rennes. His is currently the head of the Serpico (Space-timE RePresentation, Imaging and cellular dynamics of molecular COmplexes) research group. His current research interests include mathematical and statistical methods for biological image processing and microscopy. His work focuses on image sequence analysis, motion estimation, object detection, noise modeling for microscopy and traffic and dynamics modeling in cell biology. He has served as member of the program committees of the major conferences in image processing and computer vision. He is member of the editorial board of IEEE Signal Processing Letters, member of the IEEE BISP (Bio Imaging and Signal Processing) technical committee and co-head of the IPDM-BioImage Informatics node of the french national infrastructure France-BioImaging. 


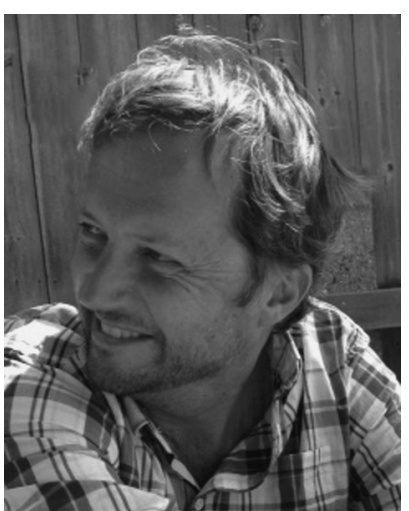

Scott T. Acton (Fellow, 2013) was born in California. He is Professor of Electrical and Computer Engineering and of Biomedical Engineering at the University of Virginia. He received his M.S. and Ph.D. degrees at the University of Texas at Austin. He received his B.S. degree at Virginia Tech. He is a Fellow of the IEEE "for contributions to biomedical image analysis."

Professor Acton's laboratory at UVA is called VIVA — Virginia Image and Video Analysis. They specialize in biological image analysis problems. The research emphases of VIVA include tracking, segmentation, representation, retrieval, classification, enhancement and image analysis problems in neuroscience. Recent theoretical interests include active contours, level sets, partial differential equation methods, scale space methods, graph signal processing and dictionary learning. Professor Acton has over 250 publications in the image analysis area including the books Biomedical Image Analysis: Tracking and Biomedical Image Analysis: Segmentation. Professor Acton currently serves as Editor-in-Chief of the IEEE Transactions on Image Processing.

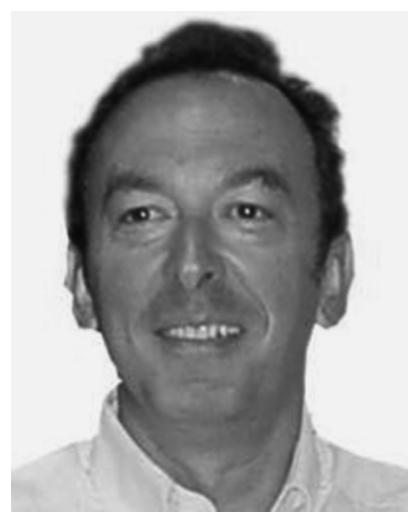

Jean-Christophe Olivo-Marin received the Ph.D. and Habilitation à Diriger des Recherches degrees in Optics and Signal Processing from the Institut d'Optique Théorique et Appliquée, University of Paris-Orsay, France. He is the head of the Bioimage Analysis Unit and the director of the Center for Innovation and Technological Research at Institut Pasteur, Paris. He has chaired the Cell Biology and Infection Department and was one of the cofounders of the Institut Pasteur Korea, Seoul, South Korea. Previous to that, he was a staff scientist at EMBL (Heidelberg). His research over the years has been centred about bioimage analysis, more specifically algorithms for multi-particle and cell tracking, microscope modelling, image deconvolution and mathematical imaging. He is a strong proponent of reproducible research, which is at the heart of the Icy software (icy.bioimageanalysis.org). He is co-head of the IPDM-BioImage Informatics node of the french national infrastructure France-BioImaging. He is a Fellow of the IEEE, an IEEE SPS Distinguished Lecturer, chair of the IEEE International Symposium on Biomedical Imaging Steering Committee, and a member of the editorial boards of IEEE Signal Processing Letters, Medical Image Analysis and BMC Bioinformatics. He was the General Chair of the 2008 IEEE International Symposium on Biomedical Imaging.

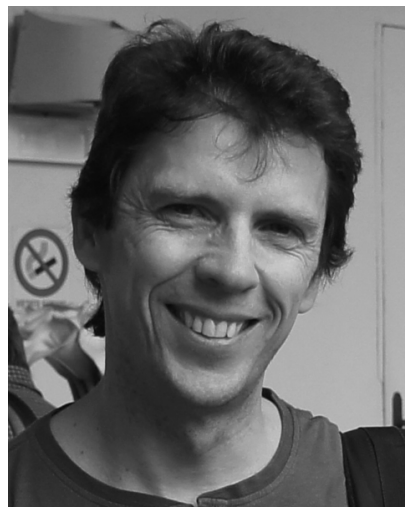

Carlos Óscar Sánchez Sorzano is B.Sc. and M. Sc. Electrical Engineering with two specialities (Electronics and Networking, Univ. Málaga), B.Sc. Computer Science (Univ. Málaga), B.Sc. and M. Sc. in Mathematics, (speciality in Statistics, UNED), Ph.D. in Biomedical Engineering (Univ. Politécnica de Madrid) and Ph.D. in Pharmacy (Univ. San Pablo-CEU). He served as secretary of the Dept. of Engineering of Electronic and Telecommunication Systems of the Univ. CEU-San Pablo (Madrid) between 2005 and 2008, coordinator of the Section on Signal and Communications theory between 2004 and 2009, head of the Bioengineering Laboratory of that University between 2007 and 2008, director of the Summerschool on Advanced Data Analysis and Modelling between 2006 and 2009, and codirector of the Master on Computational Biotechnology between 2007 and 2009. He did his $\mathrm{Ph}$.D. at the Biocomputing Unit of the National Center of Biotechnology (CSIC), and a post-doc at the Biomedical Imaging Group of the Swiss Federal Institute of Technology Lausanne (EPFL). In 2006, he received the Ángel Herrera research prize. He is senior member of the IEEE since 2008 and that same year he was accredited as "profesor titular de universidad" by ANECA. In 2009, he was appointed as "Profesor Agregado" at Univ. San Pablo CEU, awarded a Ramón y Cajal research contract and appointed as technical director of the INSTRUCT Image Processing Center for Microscopy. In 2011 and 2012, he was president of the National Association of Ramón y Cajal researchers. He coordinates the service of image processing and statistical analysis of the CNB since 2011. In 2013, he was accredited as Full Professor.

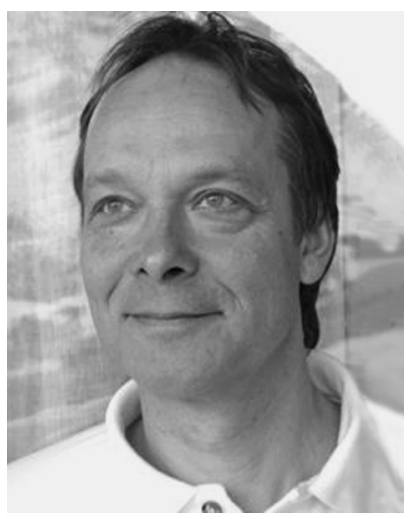

Michael Unser (M'89-SM'94-F'99) is professor and director of EPFL's Biomedical Imaging Group, Lausanne, Switzerland. His primary area of investigation is biomedical image processing. $\mathrm{He}$ is internationally recognized for his research contributions to sampling theory, wavelets, the use of splines for image processing, stochastic processes, and computational bioimaging. He has published over 250 journal papers on those topics. He is the author with P. Tafti of "An introduction to sparse stochastic processes", Cambridge University Press 2014.

From 1985 to 1997, he was with the Biomedical Engineering and Instrumentation Program, National Institutes of Health, Bethesda USA, conducting research on bioimaging.

Dr. Unser has held the position of associate Editor-in-Chief (2003-2005) for the IEEE TRANSACTIONS ON MEDICAL IMAGING. He is currently member of the editorial boards of SIAM J. Imaging Sciences, IEEE J. Selected Topics in Signal Processing, and Foundations and Trends in Signal Processing. He co-organized the first IEEE International Symposium on Biomedical Imaging (ISBI2002) and was the founding chair of the technical committee of the IEEE-SP Society on Bio Imaging and Signal Processing (BISP).

Prof. Unser is a fellow of the IEEE (1999), an EURASIP fellow (2009), and a member of the Swiss Academy of Engineering Sciences. He is the recipient of several international prizes including three IEEE-SPS Best Paper Awards and two Technical Achievement Awards from the IEEE (2008 SPS and EMBS 2010). 